\section{Mortalidade materna tardia: comparação de dois comitês de mortalidade materna no Brasil}

\author{
Late maternal mortality: comparison of maternal \\ mortality committees in Brazil
}

\begin{abstract}
Mortalidad materna tardía: comparación entre dos comités de mortalidad materna en Brasil
\end{abstract}

\section{Resumo}

Estudo de base populacional que incluiu todos os óbitos maternos tardios da casuística do Comitê de Mortalidade Materna do Município de São Paulo e do Comitê Estadual de Prevenção da Mortalidade Materna do Paraná, Brasil. Foram comparados 134 óbitos do comitê de São Paulo com os 124 do Paraná do período 2004-2013, visando a demonstrar a magnitude e as causas de mortalidade materna tardia. Os óbitos maternos tardios representaram 13,4\% do total dos óbitos maternos na casuística do comitê de São Paulo e 12,1\% na do Paraná. As causas obstétricas diretas representaram 32,1\% dos óbitos maternos tardios segundo casuística do Município de São Paulo e 42,1\% no Paraná, sendo a principal causa a cardiomiopatia no puerpério nas duas casuísticas. O óbito ocorreu entre 43 e 69 dias após o parto em 44\% dos casos em São Paulo e $39,5 \%$ no Paraná. O fator de correção para morte materna tardia foi 3,3 na casuística de São Paulo e 4,3 na do Paraná. A morte materna tardia teve relevante impacto sobre a mortalidade materna geral e a cardiomiopatia no puerpério foi a principal causa de óbito obstétrico direto tardio.

Mortalidade Materna; Morte Materna; Causas de Morte
Carlos Eduardo Pereira Vega 1

Vânia Muniz Néquer Soares 2

Acácia Maria Lourenço Francisco Nasr 2

doi: 10.1590/0102-311X00197315

\author{
Correspondência \\ C. E. P. Vega \\ Comitê de Mortalidade Materna do Município de São Paulo. \\ Rua General Jardim 36, 4o andar, São Paulo, \\ SP 01223-010, Brasil. \\ carlosvega@prefeitura.sp.gov.br \\ 1 Comitê de Mortalidade Materna do Município de São Paulo \\ São Paulo, Brasil. \\ 2 Comitê Estadual de Prevenção da Mortalidade Materna do \\ Paraná, Curitiba, Brasil.
}




\section{Introdução}

A morte materna tardia é definida como o óbito de uma mulher devido a causas obstétricas diretas ou indiretas que ocorre em um período superior a 42 dias e inferior a um ano após o fim da gravidez. A Classificação Internacional de Doenças e Problemas Relacionados à Saúde adotada no Brasil - décima revisão (CID-10, 1994) - define um único código (O96) para identificar esta ocorrência 1.

A exata dimensão da mortalidade materna tardia não é evidenciada por conta das dificuldades para a sua mensuração. A falta de informação correta sobre esse evento na declaração de óbito e por não se agregar às taxas de mortalidade materna que abrangem até 42 dias após o parto/aborto dificultam o processo de investigação e sua inclusão nos sistemas de informação 2,3.

A mortalidade materna tardia, da mesma forma que a mortalidade até os 42 dias de puerpério, reflete os riscos atribuíveis à gravidez, ao parto e ao puerpério e às condições da assistência à saúde da mulher, desde o acesso aos serviços de saúde à qualidade da assistência prestada. Sua análise é também necessária para identificar ações concretas para a redução das mortes maternas evitáveis.

Estudos sobre a magnitude da mortalidade materna tardia podem demonstrar o impacto destas mortes nas estatísticas de mortalidade materna e apontar caminhos para o seu enfrentamento com base em políticas de saúde.

Um estudo de revisão da literatura 3 sobre o fator de ajuste da mortalidade materna, para comparação internacional, identificou vinte trabalhos de diferentes países sobre subnotificação das mortes maternas que continham taxas e dados relativos à inclusão e exclusão das mortes maternas tardias. $\mathrm{O}$ percentual médio de aumento das taxas no conjunto dos estudos tendo por base a inclusão das mortes maternas tardias foi de $14 \%$, concluindo que este tipo de morte tem um alto impacto nas taxas e no fator de ajuste da mortalidade materna.

A mensuração da mortalidade materna tardia é dada pela identificação dos casos e pelo cálculo da razão de mortalidade materna tardia (RMMT), obtida pela divisão do número de mortes maternas tardias de causa direta ou indireta pelo número de nascidos vivos, segundo o local e o período estudado. Atualmente, a Organização Mundial da Saúde (OMS) determina que a RMMT deva ser calculada separadamente, podendo ser agregada à razão de mortalidade materna (RMM) até 42 dias, desde que se informe a composição de cada índice 1 .

A identificação dos óbitos maternos e o cálculo da RMMT pelos serviços de epidemiologia refletem uma pesquisa mais aprimorada dos óbitos maternos. A morte materna tardia, em grande parte, é identificada somente após a investigação dos óbitos de mulheres em idade fértil pelos serviços de vigilância epidemiológica e/ou Comitês de Mortalidade Materna 4.

Os Comitês de Mortalidade Materna são considerados as instâncias mais adequadas para apurar as circunstâncias de cada óbito materno. A implantação dos comitês municipais e estaduais no Brasil foi estabelecida pelo Ministério da Saúde com o apoio da Organização Pan-Americana da Saúde e do Fundo das Nações Unidas para a Infância no final da década de 1980. Esses comitês congregam instituições governamentais, sociedade civil organizada e universidades ligadas à área da saúde da mulher, tendo como objetivos principais a identificação da magnitude da mortalidade materna, suas causas e determinantes, além de propor e acompanhar as medidas que previnam a ocorrência destas mortes evitáveis 4 .

O presente trabalho teve como objetivo comparar a mortalidade materna tardia com base nas casuísticas dos Comitês de Mortalidade Materna do Município de São Paulo e do Estado do Paraná. A escolha desses dois comitês levou em consideração a semelhança de trabalho envolvendo a mortalidade materna, a proximidade geográfica e as características populacionais semelhantes.

\section{Metodologia}

Estudo de base populacional, descritivo, de corte transversal, que incluiu as mortes maternas tardias ocorridas no período de 2004 a 2013, subdividido em dois quinquênios. As informações foram obtidas nos bancos de dados dos Comitês de Mortalidade Materna do Município de São Paulo e do Estado do Paraná. 
No período de abrangência do estudo (2004-2013), o Município de São Paulo contava com uma população média anual de 11.159.919 habitantes, com uma média de 175.830 nascidos vivos (taxa de natalidade de 15,76 por 1.000 habitantes) e o Estado do Paraná a média anual de 10.498 .509 habitantes e 153.612 nascidos vivos (taxa de natalidade de 14,63 por 1.000 habitantes) (http://tabnet.datasus.gov. br/cgi/tabcgi.exe?ibge/cnv/poppr.def, acessado em 18/Mai/2015).

O Comitê de Mortalidade Materna do Município de São Paulo atua desde 1992, realizando a busca ativa de casos positivos ou presumíveis de morte materna por meio de metodologia própria baseada em orientações do Ministério da Saúde. Vem estabelecendo um diagnóstico real da situação da mortalidade materna baseando-se na formação de uma casuística consistente e específica para a cidade de São Paulo. Conta com equipe treinada e capacitada para desempenhar o papel de vigilância e análise dos casos de morte materna 5.

O Paraná tem um Comitê Estadual e 22 Comitês Regionais de Prevenção da Mortalidade Materna que foram implantados a partir de 1989, além dos comitês municipais de Curitiba e de outros municípios com maior população. Os óbitos maternos são identificados após a investigação dos óbitos de mulheres em idade fértil pelas equipes de vigilância epidemiológica nos 399 municípios paranaenses, sendo posteriormente revisados e analisados pelo Comitê Estadual de Prevenção da Mortalidade Materna 6.

Para a classificação de um óbito de mulher em idade fértil como morte materna tardia, os comitês procuram inicialmente estabelecer a relação causal entre a patologia que levou ao óbito com o processo gravídico-puerperal ocorrido em até um ano antes do óbito. Estabelecido o nexo, o caso é codificado como O96 (morte materna tardia) pela CID-10 e complementado na sequência com o CID da causa básica obstétrica direta ou indireta (por exemplo: O96/O90.3 ou O96/O99.4). Após a devida codificação, os municípios corrigem a causa básica no Sistema de Informações sobre Mortalidade (SIM) do Ministério da Saúde.

Em conformidade com o conceito da OMS, os óbitos acidentais ou incidentais não são considerados nem incluídos na determinação da RMMT 1 . Tal medida visa a evitar uma superenumeração do indicador, tendo em vista que os principais objetivos da identificação das mortes maternas tardias são definir medidas de prevenção relacionadas à assistência obstétrica e propor medidas sociais necessárias para evitar a ocorrência de novos óbitos.

Embora a Declaração de Óbito (DO) no Brasil tenha um campo específico para a notificação do óbito materno, a investigação detalhada de cada caso deve ser realizada com o intuito de confirmar se a informação foi corretamente assinalada, apurar com maior precisão as causas e determinantes do óbito e estabelecer se existe nexo com o ciclo gravídico-puerperal. Esse procedimento é fundamental para a garantia de estatísticas mais confiáveis 2 .

Foram analisados no presente trabalho, o número e a frequência dos óbitos maternos tardios em dois quinquênios, a causa básica dos óbitos, os dados do pré-natal e parto, o tempo transcorrido entre o parto e o óbito e a subnotificação da ocorrência. Foi calculada a RMMT e determinado o fator de correção do óbito materno tardio para este estudo.

Os bancos de dados dos dois comitês constituem fontes de dados secundários, são de domínio público e preservam o anonimato das mulheres, dos profissionais e serviços envolvidos.

\section{Resultados}

No quinquênio 2004-2008 ocorreram 507 óbitos maternos no Município de São Paulo, sendo 68 $(13,4 \%)$ considerados maternos tardios. No Estado do Paraná, foram 552 óbitos maternos sendo 67 $(12,1 \%)$ tardios. No quinquênio 2009-2013, foram identificados 476 óbitos maternos no Município de São Paulo (13,9\% tardios) e, na casuística do Paraná, 433 óbitos maternos, com 47 (10,9\%) óbitos tardios. A média da proporção das mortes maternas tardias no período de dez anos foi 13,6\% na casuística de São Paulo e 11,6\% na do Paraná (Tabela 1).

Nos 10 anos deste estudo, a RMMT foi de 7,62/100.000 nascidos vivos segundo a casuística do Município de São Paulo e de 7,42/100.000 nascidos vivos no Estado do Paraná. A RMM até 42 dias de puerpério foi de 48,29/100.000 nascidos vivos no Município de São Paulo e de 56,66/100.000 nascidos vivos no Paraná no mesmo período. 
Frequência e percentual das mortes maternas não tardias e tardias dos comitês de mortalidade materna do Município de São Paulo e do Estado do Paraná, Brasil, nos quinquênios 2004-2008 e 2009-2013.

\begin{tabular}{|c|c|c|c|c|}
\hline \multirow[t]{2}{*}{ Tipo de morte materna } & \multicolumn{2}{|c|}{ Município de São Paulo } & \multicolumn{2}{|c|}{ Estado do Paraná } \\
\hline & $\mathbf{n}$ & $\%$ & $\mathbf{n}$ & $\%$ \\
\hline \multicolumn{5}{|l|}{ 2004-2008 } \\
\hline Não tardio (até 42 dias pós-parto) & 439 & 86,6 & 485 & 87,8 \\
\hline Tardio (de 43 dias até 1 ano pós-parto) & 68 & 13,4 & 67 & 12,1 \\
\hline Subtotal & 507 & 100,0 & 552 & 100,0 \\
\hline \multicolumn{5}{|l|}{$2009-2013$} \\
\hline Não tardio (até 42 dias pós-parto) & 410 & 86,1 & 386 & 89,1 \\
\hline Tardio (de 43 dias até 1 ano pós-parto) & 66 & 13,9 & 47 & 10,9 \\
\hline Subtotal & 476 & 100,0 & 433 & 100,0 \\
\hline \multicolumn{5}{|l|}{ 2004-2013 } \\
\hline Não tardio (até 42 dias pós-parto) & 849 & 86,4 & 871 & 88,4 \\
\hline Tardio (de 43 dias até 1 ano pós-parto) & 134 & 13,6 & 114 & 11,6 \\
\hline Total & 983 & 100,0 & 985 & 100,0 \\
\hline
\end{tabular}

A razão de mortalidade materna total (RMM Total), com a inclusão das mortes maternas tardias, aumentou em 15,8\% no Município de São Paulo e em 13,1\% no Estado do Paraná, chegando a 55,91 e $64,08 / 100.000$ nascidos vivos, respectivamente, no período estudado (Tabela 2).

$\mathrm{Na}$ análise da causa básica das mortes maternas tardias evidencia-se que as causas obstétricas diretas representaram 32,1\% das mortes tardias e as causas indiretas 66,4\% no Município de São Paulo. No Estado do Paraná foram, respectivamente, 42,1\% e 52,6\%. Nos dois comitês, a cardiomiopatia no puerpério (CMPP) foi a principal causa de óbito obstétrico direto tardio. Como causas secundárias, identificamos as complicações de aborto e a infecção puerperal no Município de São Paulo e a pré-eclâmpsia/eclâmpsia e complicações do parto no Estado do Paraná. Dentre as causas obstétricas indiretas tardias, em ambos os comitês, predominaram os óbitos por neoplasias (15,7\% no Município de São Paulo e 12,3\% no Estado do Paraná) seguidas pelas doenças do aparelho circulatório (14,9\% e $9,6 \%$, respectivamente).

Em 1,5\% dos casos de mortes maternas tardias ocorridas no Município de São Paulo e em 5,3\% dos casos no Estado do Paraná não foi possível determinar a causa básica do óbito (CID O96/O95) (Tabela 3).

Os dados da Tabela 4 demonstram que 59,7\% das mulheres realizaram 4 ou mais consultas de pré-natal no Município de São Paulo e 72,8\% no Estado do Paraná. O estudo identificou que 15,7\% e $3,5 \%$ das falecidas não realizaram o pré-natal, respectivamente nos dois comitês. A cesariana foi a via de parto utilizada em 50,7\% dos casos no Município de São Paulo contra 42,1\% no Estado do Paraná. Ainda na Tabela 4, a maior parte dos óbitos ocorreu do $43^{\circ}$ ao $99^{\circ}$ dia de puerpério, sendo predominantemente dentro de um ambiente hospitalar nos dois locais estudados.

Referente à notificação ou declaração das mortes maternas tardias na declaração de óbito pelos médicos (Tabela 5), o Município de São Paulo identificou que, das 134 mortes maternas tardias, 94 (70,1\%) não estavam declaradas. Na casuística do Paraná, das 114 mortes, 88 (77,2\%) não estavam declaradas e foram identificadas apenas após a investigação dos óbitos de mulheres em idade fértil pelos serviços de vigilância epidemiológica dos municípios, gerando um fator de correção de 3,4 no Município de São Paulo e de 4,3 no Estado do Paraná.

Se considerados os campos específicos da DO que poderiam identificar as mortes maternas após 43 dias até um ano após o parto, evidenciamos que 53,7\% estavam assinalados positivamente no Município de São Paulo. No Estado do Paraná, 58,8\% tinham esse campo preenchido, denotando a subnotificação e a necessidade de investigação detalhada dos óbitos das mulheres em idade fértil para a identificação das mortes maternas tardias e não tardias. 
Tabela 2

Razão de Mortalidade Materna (RMM), por 100.000 nascidos vivos, dos comitês de mortalidade materna do Município de São Paulo e do Estado do Paraná, Brasil, 2004-2008 e 2009-2013.

\begin{tabular}{lccc}
\hline & \multicolumn{3}{c}{ RMM } \\
& $\mathbf{2 0 0 4 - 2 0 0 8}$ & $\mathbf{2 0 0 9 - 2 0 1 3}$ & Total (2004-2013) \\
\hline Município de São Paulo & & & \\
Não tardia & 49,75 & 46,81 & 48,29 \\
Tardia & 7,71 & 7,54 & 7,62 \\
Total & 57,46 & 54,35 & 55,91 \\
Estado do Paraná & & & 56,66 \\
Não tardia & 62,81 & 50,50 & 7,42 \\
Tardia & 8,68 & 6,20 & 64,08 \\
Total & 71,49 & 56,70 & \\
\hline
\end{tabular}

Tabela 3

Causa básica das mortes maternas tardias segundo casuísticas dos comitês de mortalidade materna do Município de São Paulo e do Estado do Paraná, Brasil, 2004-2008 e 2009-2013.

\begin{tabular}{|c|c|c|c|c|c|c|c|c|}
\hline \multirow[t]{2}{*}{ Causa básica } & \multicolumn{4}{|c|}{ Município de São Paulo } & \multicolumn{4}{|c|}{ Estado do Paraná } \\
\hline & 2004-2008 & 2009-2013 & Total & $\%$ & 2004-2008 & 2009-2013 & Total & $\%$ \\
\hline Obestétricas diretas & 21 & 22 & 43 & 32,1 & 24 & 24 & 48 & 42,1 \\
\hline Aborto & 4 & 4 & 8 & 6,0 & 3 & 0 & 3 & 2,6 \\
\hline Pré-eclâmpsia/Eclâmpsia & 1 & 3 & 4 & 3,0 & 5 & 6 & 11 & 9,6 \\
\hline $\begin{array}{l}\text { Complicações do parto (rotura uterina, } \\
\text { hemorragia, traumatismo) }\end{array}$ & 3 & 0 & 3 & 2,2 & 3 & 4 & 7 & 6,1 \\
\hline Complicações anestésicas no parto & 1 & 0 & 1 & 0,7 & 2 & 0 & 2 & 1,8 \\
\hline Infecção urinária e renal na gravidez & 1 & 1 & 2 & 1,5 & 1 & 1 & 2 & 1,8 \\
\hline Tromboembolia & 0 & 0 & 0 & 0,0 & 1 & 0 & 1 & 0,9 \\
\hline Infecção puerperal & 4 & 2 & 6 & 4,5 & 3 & 3 & 6 & 5,3 \\
\hline Cardiomiopatia no puerpério & 6 & 9 & 15 & 11,2 & 6 & 7 & 13 & 11,4 \\
\hline Outras diretas & 1 & 3 & 4 & 3,0 & 0 & 3 & 3 & 2,6 \\
\hline Obstétricas indiretas & 46 & 43 & 89 & 66,4 & 38 & 23 & 61 & 53,5 \\
\hline Doenças do aparelho circulatório & 6 & 14 & 20 & 14,9 & 7 & 4 & 11 & 9,6 \\
\hline Hipertensão arterial crônica & 7 & 3 & 10 & 7,5 & 2 & 1 & 3 & 2,6 \\
\hline Doenças do aparelho respiratório & 2 & 2 & 4 & 3,0 & 5 & 3 & 8 & 7,0 \\
\hline Doenças do aparelho digestivo & 1 & 3 & 4 & 3,0 & 3 & 2 & 5 & 4,4 \\
\hline Doenças infecciosas & 5 & 0 & 5 & 3,7 & 4 & 2 & 6 & 5,3 \\
\hline $\begin{array}{l}\text { Neoplasias (mama, gástrico, útero, ovário, } \\
\text { linfoma) }\end{array}$ & 12 & 9 & 21 & 15,7 & 8 & 6 & 14 & 12,3 \\
\hline Lupus & 3 & 2 & 5 & 3,7 & 3 & 1 & 4 & 3,5 \\
\hline HIV & 2 & 1 & 3 & 2,2 & 2 & 1 & 3 & 2,6 \\
\hline Outras indiretas & 8 & 9 & 17 & 12,7 & 4 & 3 & 7 & 6,1 \\
\hline Causas maternas indeterminadas & 1 & 1 & 2 & 1,5 & 5 & 0 & 5 & 4,4 \\
\hline Total & 68 & 66 & 134 & 100,0 & 67 & 47 & 114 & 100,0 \\
\hline
\end{tabular}


Tabela 4

Mortes maternas tardias segundo a atenção obstétrica - casuísticas dos comitês de mortalidade materna do Município de São Paulo e do Estado do Paraná, Brasil, 2004-2008 e 2009-2013.

\begin{tabular}{|c|c|c|c|c|c|c|c|c|}
\hline \multirow[t]{2}{*}{ Variável } & \multicolumn{4}{|c|}{ Município de São Paulo } & \multicolumn{4}{|c|}{ Estado do Paraná } \\
\hline & 2004-2008 & $2009-2013$ & Total & $\%$ & 2004-2008 & 2009-2013 & Total & $\%$ \\
\hline \multicolumn{9}{|l|}{ Consultas de pré-natal } \\
\hline Até 4 & 13 & 12 & 25 & 18,7 & 8 & 9 & 17 & 14,9 \\
\hline Mais que 4 & 41 & 39 & 80 & 59,7 & 48 & 35 & 83 & 72,8 \\
\hline Não realizou & 8 & 13 & 21 & 15,7 & 3 & 1 & 4 & 3,5 \\
\hline Ignorado & 6 & 2 & 8 & 6,0 & 8 & 2 & 10 & 8,8 \\
\hline \multicolumn{9}{|l|}{ Tipo de parto } \\
\hline Normal & 22 & 30 & 52 & 38,8 & 31 & 31 & 62 & 54,4 \\
\hline Cesariana & 36 & 32 & 68 & 50,7 & 34 & 14 & 48 & 42,1 \\
\hline Pós-aborto & 7 & 3 & 10 & 7,5 & 2 & 1 & 3 & 2,5 \\
\hline Ignorado & 3 & 1 & 4 & 3,0 & 0 & 1 & 1 & 0,9 \\
\hline \multicolumn{9}{|l|}{ Tempo entre o parto e o óbito (dias) } \\
\hline $43-69$ & 33 & 26 & 59 & 44,0 & 25 & 20 & 45 & 39,5 \\
\hline $70-99$ & 13 & 10 & 23 & 17,2 & 20 & 9 & 29 & 25,4 \\
\hline $100-199$ & 15 & 19 & 34 & 25,4 & 10 & 13 & 23 & 20,2 \\
\hline 200 ou mais & 5 & 11 & 16 & 11,9 & 12 & 4 & 16 & 14,0 \\
\hline Ignorado & 2 & 0 & 2 & 1,5 & 0 & 1 & 1 & 0,9 \\
\hline \multicolumn{9}{|l|}{ Local do óbito } \\
\hline Hospitalar & 67 & 65 & 132 & 98,5 & 51 & 41 & 92 & 80,7 \\
\hline Outros estabelecimentos de saúde & 0 & 0 & 0 & 0,0 & 15 & 1 & 16 & 14,0 \\
\hline Outros & 1 & 1 & 2 & 1,5 & 1 & 5 & 6 & 5,3 \\
\hline Total & 68 & 66 & 134 & 100,0 & 67 & 47 & 114 & 100,0 \\
\hline
\end{tabular}

\section{Tabela 5}

Subnotificação e fator de correção (FC) da morte materna tardia - casuística do Comitê de Mortalidade Materna do Município de São Paulo comparada à casuística do Comitê Estadual de Prevenção da Mortalidade Materna do Estado do Paraná por quinquênio. Brasil, 2004-2008 e 2009-2013.

\begin{tabular}{|c|c|c|c|c|c|c|c|c|c|c|c|c|}
\hline \multirow[t]{3}{*}{ Variável } & \multicolumn{6}{|c|}{ Município de São Paulo } & \multicolumn{6}{|c|}{ Estado do Paraná } \\
\hline & \multicolumn{2}{|c|}{ Sim } & \multicolumn{2}{|c|}{ Não } & \multirow[t]{2}{*}{ Total } & \multirow[t]{2}{*}{ FC } & \multicolumn{2}{|c|}{ Sim } & \multicolumn{2}{|c|}{ Não } & \multirow[t]{2}{*}{ Total } & \multirow[t]{2}{*}{ FC } \\
\hline & $\mathbf{n}$ & $\%$ & $\mathbf{n}$ & $\%$ & & & $\mathbf{n}$ & $\%$ & $\mathbf{n}$ & $\%$ & & \\
\hline \multicolumn{13}{|c|}{ Mortalidade materna tardia declarada na DO } \\
\hline 2004-2008 & 18 & 26,5 & 50 & 73,5 & 68 & 3,8 & 14 & 20,9 & 53 & 79,1 & 67 & 4,8 \\
\hline $2009-2013$ & 22 & 33.3 & 44 & 66,7 & 66 & 3,0 & 12 & 25,5 & 35 & 74,5 & 47 & 3,9 \\
\hline Total & 40 & 29,9 & 94 & 70,1 & 134 & 3,4 & 26 & 22,8 & 88 & 77,2 & 114 & 4,4 \\
\hline \multicolumn{13}{|c|}{ Preenchimento do campo $44 / 37$ * } \\
\hline $2004-2008$ & 37 & 54,4 & 31 & 45,6 & 68 & - & 36 & 53,7 & 31 & 46,3 & 67 & - \\
\hline $2009-2013$ & 35 & 53,0 & 31 & 47,0 & 66 & - & 31 & 66,0 & 16 & 34,0 & 47 & - \\
\hline Total & 72 & 53,7 & 62 & 46,3 & 134 & - & 67 & 58,8 & 47 & 41,2 & 114 & - \\
\hline
\end{tabular}

DO: Declaração de Óbito.

* Campo 44 na DO antiga e campo 37 na atual. 
As causas básicas na DO original, que ocultavam as mortes maternas tardias no Paraná, eram: cardiopatia, insuficiência cardíaca congestiva, infarto agudo do miocárdio, insuficiência respiratória, tuberculose, pneumonia, embolia pulmonar, asma e neoplasias (gástrico, ovário, mama, útero). No Município de São Paulo as causas que mais frequentemente ocultavam as mortes maternas tardias foram as doenças do aparelho circulatório, seguidas das neoplasias.

\section{Discussão}

A morte materna tardia é enquadrada no código genérico O96 conforme a CID-10 vigente desde 1994 no Brasil, ou seja, não existe a possibilidade de especificação das causas obstétricas diretas ou indiretas que levaram ao óbito, como também não são incluídas no cálculo da RMM. Por esse motivo, é ainda subestimada, pouco visibilizada e pouco estudada no Brasil e em outros países 1,2,3.

No presente trabalho, evidenciou-se que as mortes maternas tardias apresentaram alta magnitude e resultado semelhante nas duas casuísticas nos dois quinquênios estudados. O percentual de aumento nas taxas (RMM Total) com a inclusão dos óbitos maternos tardios no cálculo foi de 15,8\% no Município de São Paulo e 13,1\% no Paraná.

Em um estudo de revisão da literatura que incluiu vinte trabalhos do Reino Unido, Estados Unidos, Argentina, México, Brasil, Austrália, Áustria, Canadá, Dinamarca, Finlândia, Geórgia, Eslovênia e Suécia, a diferença percentual média para o fator de ajuste com a inclusão das mortes maternas tardias foi de $14 \%$, concluindo que estas mortes são consideradas nos registros e nos estudos sobre mortalidade materna em diferentes países e impactam nas taxas e fator de ajuste da mortalidade materna ${ }^{3}$.

Um estudo realizado na Austrália 7 no período de 1994 a 2001, considerou 76 mortes maternas tardias $(43,9 \%)$ dentre o total de 173 relatadas, sendo que as principais causas foram o suicídio (23 casos), os distúrbios cardíacos (16 casos), e acidentes/violência (16 casos). Nesse estudo, foram incluidos os óbitos incidentais e acidentais ou não obstétricos (suicídio, acidentes e violência), diferentemente do preconizado pela OMS, determinando uma proporção muito superior das mortes maternas tardias naquele país.

No Brasil, estudo realizado nas capitais brasileiras em 20028 identificou 13,8\% de mortes maternas tardias (33 dos 239 óbitos maternos confirmados). Os resultados da investigação dos óbitos tardios nesse estudo apontaram como a causa obstétrica de maior ocorrência a CMPP (24,2\%), seguida da eclâmpsia/pré-eclâmpsia $(21,3 \%)$ e das doenças do aparelho circulatório complicando a gravidez $(12,1 \%)$.

Concordante com a pesquisa mencionada anteriormente, a principal causa de morte materna tardia obstétrica direta identificada em ambas as casuísticas do presente estudo foi também a CMPP. Em sequência, o comitê do Paraná identificou as síndromes hipertensivas (pré-eclâmpsia/eclâmpsia) e as complicações do parto (rotura uterina, hemorragia, tocotraumatismo). O Município de São Paulo constatou que as complicações de aborto e a infecção puerperal foram sucedidas à causa principal. A infecção puerperal como causa de morte foi semelhante nas duas casuísticas analisadas.

As causas das mortes maternas tardias encontradas poderiam ser evitadas considerando que existem tratamentos específicos efetivos para todas elas, apontando a necessidade de acompanhamento mais prolongado no puerpério, especialmente nos casos de maior risco, proporcionando tratamento adequado e em tempo hábil.

Medidas simples, mas importantes, como o planejamento reprodutivo, monitoramento e tratamento das cardiopatias no puerpério, sulfato de magnésio na pré-eclâmpsia e eclâmpsia, antibióticos na infecção, abortamento seguro, ocitocina ou misoprostol na hemorragia pós-parto e capacitação profissional contribuiriam para a redução das causas identificadas, garantindo assim o direito à vida dessas mulheres 9 .

A CMPP identificada como a primeira causa de morte materna tardia, deve ser encarada como um evento sentinela no puerpério e, portanto, alguns aspectos desta patologia foram destacados com base na literatura.

A CMPP é um tipo de cardiomiopatia dilatada, que ocorre em mulheres previamente saudáveis, no período entre o último mês de gravidez e os cinco meses após o parto 10. 
A etiologia de CMPP permanece mal definida e muitas causas têm sido sugeridas: miocardite viral, resposta imune anormal à gravidez, resposta inadequada a estresses hemodinâmicos da gravidez, citocinas ativadas pelo estresse, distúrbios hormonais, uso prolongado de tocolíticos, hereditariedade e déficits nutricionais 11 .

Os fatores de risco para a CMPP incluem multiparidade, idade materna avançada, gravidez multifetal, pré-eclâmpsia e hipertensão gestacional. Não está claro se a raça representa um fator de risco independente ou se é a interação decorrida com hipertensão que aumenta o risco de CMPP 12. Mulheres com esses fatores deveriam ser monitoradas no puerpério.

Os critérios diagnósticos de CMPP são: insuficiência cardíaca no último mês de gestação ou nos primeiros cinco meses após o parto de etiologia desconhecida, ausência de cardiopatia prévia ou disfunção sistólica documentada 13. Um ecocardiograma (ECG) deve ser realizado em todas as pacientes com suspeita de CMPP. Os critérios ecográficos para o seu diagnóstico são: fração de ejeção menor que $45 \%$, encurtamento percentual menor que 30\%, diâmetro diastólico final do ventrículo esquerdo maior que $2,7 \mathrm{~cm} / \mathrm{m}^{2} 12,14$.

Os sinais e sintomas da CMPP são semelhantes aos que aparecem em cardiopatias, sendo que os sintomas precoces podem ser semelhantes às alterações fisiológicas do final da gestação como dispneia, fadiga, edema de membros inferiores, ortopneia, dispneia paroxística noturna e tosse persistente. Na CMPP também estão comumente presentes dor abdominal, tonturas, dor precordial e palpitações, e nas fases posteriores, a hipotensão postural pode ocorrer ${ }^{15}$.

O tratamento clínico da CMPP é similar ao de outras formas de insuficiência cardíaca decorrente de disfunção sistólica, com exceção de que em mulheres que apresentam durante a gravidez, os efeitos adversos potenciais para o feto devem ser considerados. A terapia com inibidor da enzima conversora da angiotensina e antagonista do receptor de angiotensina é contraindicada durante a gravidez 16.

O parto vaginal espontâneo é preferível em mulheres com função cardíaca controlada e feto com boa vitalidade, e a cesariana planejada é preferível para as mulheres que estão gravemente doentes e precisam de terapia inotrópica ou mecânica, além de suporte cardiovascular durante o trabalho de parto. A avaliação cuidadosa dos fatores de risco na gestação pode ajudar na prevenção da CMPP, assim como a estratificação por risco das mulheres que se recuperaram de CMPP é necessária para prever o risco de futuras gestações 15 .

Dentre as causas obstétricas indiretas, os óbitos por neoplasias foram relevantes entre os casos de mortes maternas tardias. O câncer associado à gravidez é raro, afeta aproximadamente 1 em 1.000 gravidezes, mas 20 a 30\% das mulheres que recebem o diagnóstico de câncer estão em idade reprodutiva 17 .

Os principais tipos de neoplasia que acometem a mulher na idade reprodutiva são o de mama, o do colo do útero, o linfoma, o câncer de ovário, e o melanoma, embora o câncer durante a gravidez possa surgir a partir de qualquer local 18 .

No acompanhamento pré-natal, a avaliação da gestante deve incluir história e exame físico completo, contemplando o exame das mamas e a colpocitologia oncótica para o diagnóstico precoce, desencadeando as intervenções necessárias 19.

O diagnóstico de câncer na gravidez é mais difícil em face de fatores como: (1) sobreposição dos sintomas de malignidade com os sintomas da gravidez como náuseas, vômitos, alterações da mama e dor abdominal; (2) exame físico comprometido devido a alterações da mama e útero gravídico; (3) hesitação para realizar determinados exames e as preocupações com imagem corporal; (4) diminuição da utilidade dos marcadores tumorais e valores laboratoriais neste período 20.

O tratamento do câncer na gravidez deve ser realizado por equipe multidisciplinar e tem por objetivo otimizar o tratamento materno, minimizando os danos ao feto. Entretanto, esse tratamento depende da extensão da doença, das opções de tratamento necessárias, do impacto sobre a gravidez, bem como da idade gestacional no momento do diagnóstico 21,22,23.

A população deve ser conscientizada sobre a necessidade de um acompanhamento de saúde de forma rotineira visando ao diagnóstico precoce do câncer, e a classe médica deve ser orientada sobre os procedimentos de diagnóstico e tratamento de uma gestante com câncer 22,23.

Para identificar aspectos da qualidade da atenção obstétrica nos casos dos óbitos maternos tardios, o número de consultas de pré-natal, tipo de parto, local do óbito e tempo transcorrido entre o parto e o óbito foram levados em consideração. 
Promover a saúde materna engloba, dentre outros aspectos, a realização do mínimo preconizado de consultas de pré-natal com qualidade e o estabelecimento de diagnóstico e tratamento das doenças intercorrentes da gestação ${ }^{9}$. Doenças cardíacas, neoplasias e outras intercorrências devem ser precocemente identificadas e tratadas no pré-natal em serviços especializados, evitando complicações no parto e no puerpério 9,24 .

Identificou-se que $30 \%$ dos casos realizaram menos de quatro consultas ou não realizaram o prénatal, mas a maioria teve número superior a quatro consultas. No Brasil, o Programa Nacional de Humanização do Pré-natal e Nascimento (PNHPN) estabelece no mínimo seis consultas de pré-natal, preferencialmente, com uma no primeiro trimestre, duas no segundo e três no último trimestre 9 .

Práticas realizadas rotineiramente durante a assistência pré-natal estão associadas a melhores desfechos perinatais. Segundo recomendações do Ministério da Saúde, a assistência pré-natal deve se dar por meio da incorporação de condutas acolhedoras; do desenvolvimento de ações educativas e preventivas, sem intervenções desnecessárias; da detecção precoce de patologias e de situações de risco gestacional; do estabelecimento de vínculo entre o pré-natal e o local do parto; e do fácil acesso a serviços de saúde de qualidade, desde o atendimento ambulatorial básico até o atendimento hospitalar voltado à gestação de alto risco. Amplo estudo multicêntrico nacional demonstrou a existência de falhas na assistência pré-natal, incluindo dificuldades no acesso, início tardio, número inadequado de consultas e realização incompleta dos procedimentos preconizados, afetando sua qualidade e efetividade 9,24 .

Outro indicador da atenção obstétrica ao parto, a taxa de cesariana, neste estudo extrapola o parâmetro de 15\% recomendado pela OMS. A taxa de cesárea continua aumentando no nosso país, embora o procedimento esteja associado à maior permanência hospitalar, à maior morbidade respiratória perinatal, à maior incidência de complicações em futuras gestações, além de onerar o sistema de saúde. A proporção de cesariana cresceu de 38\% em 2000 para 52\% em 2010, elevação associada às mudanças nas práticas obstétricas, não necessariamente relacionadas a razões clínicas, elevando o risco de morbimortalidade, como demonstrado e debatido em estudos recentes 25,26.

O tempo decorrido entre o parto e o óbito foi na maioria dos casos de aproximadamente dois meses, nas duas casuísticas. O Ministério da Saúde preconiza duas consultas após o parto, recomendando uma visita domiciliar na primeira semana após a alta do bebê (Primeira Semana de Saúde Integral), considerando que a atenção à mulher e ao recém-nascido no pós-parto imediato e nas primeiras semanas é fundamental para a saúde materno-infantil. Na segunda consulta de puericultura deve ser realizada nova avaliação das condições da saúde da mulher e do recém-nascido; o registro das alterações; investigação e registro da amamentação; retorno da menstruação e atividade sexual; realização das ações educativas e tratamento das possíveis intercorrências 9.

Levando-se em consideração que a gravidez, o parto e o puerpério são processos fisiológicos, a maioria das mulheres que são atendidas nos serviços obstétricos necessita de cuidados básicos. Contudo, na área obstétrica podem ocorrer emergências que costumam ser repentinas e que devem ter respostas rápidas e adequadas 9 . Daí a importância de se implantar a vigilância domiciliar quinzenal, por período de até dois meses após o parto, para as gestantes e puérperas de risco, devido ao elevado número de mortes maternas tardias, procedendo ao rápido encaminhamento e tratamento das complicações deste período.

Quanto ao local do óbito, verificou-se que um maior número de casos ocorreu fora do ambiente hospitalar no Paraná em relação à casuística de São Paulo, indicando a necessidade de se avaliar o motivo desta situação: dificuldade no acesso; deficiência no atendimento e diagnóstico de doenças que surgiram ou agravaram na gestação; acompanhamento da mulher no ciclo gravídico-puerperal, que encerrou na consulta de puerpério, quando o monitoramento deveria se estender, pelo menos, até o 42 o dia após o parto.

Uma equipe multiprofissional treinada e capacitada, disponibilidade de equipamentos, banco de sangue, infraestrutura laboratorial adequada ao diagnóstico e tratamento dos casos das gestações de alto risco são condições relacionadas a um desfecho favorável nas complicações obstétricas. A oferta de atenção qualificada aparece como um componente essencial para a redução da mortalidade materna 24.

A evolução científica e tecnológica nas últimas décadas resultou em benefícios na área de saúde materna e fetal, avanços estes que permitiriam evitar a quase totalidade das mortes maternas e diminuir consideravelmente as patologias relacionadas com a reprodução. 
Verificou-se a alta vulnerabilidade dos óbitos maternos tardios nas duas casuísticas estudadas, ao identificar que $40 \%$ dos óbitos eram devidos a causas obstétricas diretas, que poderiam ser evitados por uma assistência obstétrica mais qualificada. Já os óbitos obstétricos indiretos que corresponderam a cerca de $60 \%$, poderiam ser reduzidos pelo controle das doenças crônicas como as neoplasias, cardiopatias, hipertensão e/ou manejo adequado destas patologias em mulheres grávidas ou puérperas.

A busca ativa e o monitoramento da saúde envolvendo as puérperas de risco, até um ano após o término da gravidez nos diversos níveis de atendimento, poderão contribuir para a redução das taxas de mortalidade materna tardia. É imprescindível a formalização das referências hospitalares resolutivas para puérperas que apresentaram gestação de alto risco até um ano após o parto.

Por outro lado, quando ocorre o óbito na fase tardia do puerpério é frequente a subnotificação e o preenchimento incorreto das DO, o que implica falhas na análise final da causa mortis e, consequentemente, na definição de estratégias para a redução das mortes maternas 2 . A morte materna tardia ficaria oculta se não houvesse o trabalho de investigação das vigilâncias epidemiológicas dos municípios e análise e divulgação da sua magnitude e gravidade, pelos comitês de mortalidade materna.

A subnotificação das mortes maternas tardias é maior do que as ocorridas até 42 dias do puerpério. A vigilância epidemiológica da morte materna tardia deve ser incentivada no país para a obtenção de um perfil mais realista desta mortalidade em cada estado, com vistas à comparação destes dados com os avanços da assistência obstétrica e da saúde materna no Brasil.

Um estudo realizado no Brasil em 2014 27, com o objetivo de propor uma metodologia de estimativa da razão de mortalidade materna no Brasil por meio de informações do Ministério da Saúde identificou que dos 112.007 (55,8\%) óbitos de mulheres em idade fértil investigados pelos estados houve uma reclassificação ou correção da subnotificação das mortes maternas em 39,48\% das mortes de mulheres em idade fértil com causas presumíveis de mortes maternas, em 20,96\% das mortes sem causas presumíveis de morte materna e em 11,29\% das mortes maternas tardias. Após a correção da subnotificação das mortes maternas, levando-se em conta essas proporções e a correção do sub-registro de óbitos e do número de nascidos vivos, a RMM estimada para o período foi de 68,2/100.000 nascidos vivos no Brasil, considerada ainda bastante elevada pelos autores.

Nas casuísticas do Município de São Paulo e do Paraná, a correção de subnotificação do óbito materno tardio foi bem superior: 70\% dos casos não estavam declarados, sendo reclassificados após investigação e análise pelos comitês, determinando um fator de correção de 3,4 em São Paulo e 4,4 no Paraná. A maior proporção de correção está provavelmente associada à maior investigação desses óbitos em São Paulo e no Paraná, já demonstrada em outros estudos 28,29. Os campos específicos para identificação das mortes tardias na DO estavam subpreenchidos em torno de $40 \%$ dos casos, confirmando, assim, a necessidade de investigação de todos os óbitos de mulheres em idade fértil, levandose em consideração a existência de história de gestação no ano anterior ao óbito e a sua evolução no puerpério, identificando as causas do óbito e os determinantes associados.

A recomendação do Centro Brasileiro de Classificação de Doenças para a ampliação dos códigos referentes aos óbitos maternos tardios (O96) na CID foi efetivada pela OMS na revisão da CID-10, e passou a incluir os códigos O96.0 - óbito materno tardio obstétrico direto; O96.1 - óbito materno obstétrico indireto e O96.9 - para causas indefinidas 30. A adoção dessas codificações no Brasil é, portanto, essencial para dar maior visibilidade aos óbitos maternos tardios.

A OMS busca, com a organização da CID, desenvolver uma classificação útil para a saúde pública global. O presente trabalho incorpora mais informações e conhecimento sobre mortalidade materna tardia e reforça a importância do novo sistema de classificação da CID ao incluir códigos adicionais para os óbitos maternos tardios, refletindo assim a realidade clínica destes óbitos com vistas à redução das mortes maternas em nosso país e ao redor do mundo.

A correção da subnotificação desses óbitos tardios e o cálculo da RMMT deveriam ser obrigatórios para todas as regiões e países, valorizando-se o seu impacto na mortalidade materna em geral.

A precoce identificação e tratamento dos casos de CMPP, bem como a prevenção e manejo correto das neoplasias na gestação e em mulheres em idade fértil implicariam a redução dos óbitos maternos tardios evitáveis no Brasil.

Durante a Assembleia Geral das Nações Unidas de 2015, em Nova York (Estados Unidos), a Organização das Nações Unidas e a OMS lançaram a Estratégia Global para Saúde da Mulher, Criança e do Adolescente, 2016-2030, como parte da programação dos Objetivos de Desenvolvimento Sustentável. 
Essa iniciativa dá continuidade aos Objetivos de Desenvolvimento do Milênio (1990-2015), e tem como focos eliminar todas as mortes evitáveis de mulheres, crianças e adolescentes, além de criar um ambiente em que estes grupos não apenas sobrevivam (survive), mas prosperem (thrive), transformando (transform) os seus ambientes, sua saúde e seu bem-estar. Devem ser incluídas nesses objetivos as mortes maternas tardias evitáveis 31 .

\section{Colaboradores}

Os três autores participaram de todas as fases de produção do artigo.

\section{Referências}

1. Organização Mundial da Saúde. Classificação estatística internacional de doenças e problemas relacionados à saúde - décima revisão. 3a Ed. São Paulo: Edusp; 1996.

2. Laurenti R, Melo Jorge M, Gotlieb S. Mortes maternas e mortes por causas maternas. Epidemiol Serv Saúde 2008; 17:283-92.

3. Jentoft S, Nielsen V, Roll-Hansen D. Adjusting maternal mortality data for international comparisons: the case of vital registration systems. Statistics Norway, Discussion Papers 2014; (773). https://www.ssb.no/en/forskning/ discussion-papers/_attachment/168855?_ ts $=144 \mathrm{de} 574468$.

\section{Agradecimentos}

Agradecemos aos comitês do Município de São Paulo e do Estado do Paraná pela cessão dos dados para a realização deste trabalho.
4. Ministério da Saúde. Guia de vigilância epidemiológica do óbito materno: normas e manuais técnicos. Brasília: Ministério da Saúde; 2009.

5. Vega CEP. Relatórios de mortalidade materna do município de São Paulo. http://www.pre feitura.sp.gov.br/cidade/secretarias/saude/sau de_da_mulher/index.php? $\mathrm{p}=5778$.

6. Comitê Estadual de Mortalidade Materna do Paraná. Relatório de resultados "20 anos de investigação e análise”. Mortalidade materna no Paraná 1989-2008. Curitiba: Secretaria Estadual de Saúde; 2009. 
7. Cliffe S, Black D, Bryant J, Sullivan E. Maternal deaths in New South Wales, Australia: a data linkage project. Aust N Z J Obstet Gynaecol 2008; 48:255-60.

8. Laurenti R, Mello Jorge MHP, Gotlieb SLD. A mortalidade materna nas capitais brasileiras: algumas características e estimativa de um fator de ajuste. Rev Bras Epidemiol 2004; 7:449-60.

9. Secretaria de Atenção à Saúde, Ministério da Saúde. Pré-natal e puerpério - atenção qualificada e humanizada. Brasília: Ministério da Saúde; 2006.

10. Johnson-Coyle L, Jensen L, Sobey A; American College of Cardiology Foundation; American Heart Association. Peripartum cardiomyopathy: review and practice guidelines. Am J Crit Care 2012; 21:89-98.

11. Ntusi NB, Mayosi BM. Aetiology and risk factors of peripartum cardiomyopathy: a systematic review. Int J Cardiol 2009; 131:168-79.

12. Pearson GD, Veille JC, Rahimtoola S, Hsia J, Oakley CM, Hosenpud JD, et al. Peripartum cardiomyopathy: National Heart, Lung, and Blood Institute and Office of Rare Diseases (National Institutes of Health) workshop recommendations and review. JAMA 2000; 283:1183-8.

13. Givertz MM. Cardiology patient page: peripartum cardiomyopathy. Circulation 2013; 127:e622-6.

14. Kolte D, Khera S, Aronow WS, Palaniswamy C, Mujib M, Ahn C, et al. Temporal trends in incidence and outcomes of peripartum cardiomyopathy in the United States: a nationwide population-based study. J Am Heart Assoc 2014; 3:e001056.

15. Sliwa K, Hilfiker-Kleiner D, Petrie MC, Mebazaa A, Pieske B, Buchmann E, et al. Current state of knowledge on aetiology, diagnosis, management, and therapy of peripartum cardiomyopathy: a position statement from the Heart Failure Association of the European Society of Cardiology Working Group on peripartum cardiomyopathy. Eur J Heart Fail 2010; 12:767-78.

16. Mishra N, Mishra VN, Devanshi. Peripartum cardiomyopathy. J Assoc Physicians India 2013; 61:268-73.

17. Siegel R, Naishadham D, Jemal A. Cancer statistics, 2013. CA Cancer J Clin 2013; 63:11-30.

18. Pavlidis NA. Coexistence of pregnancy and malignancy. Oncologist 2002; 7:279-87.

19. Massad LS, Einstein MH, Huh WK, Katki HA, Kinney WK, Schiffman M, et al. 2012 updated consensus guidelines for the management of abnormal cervical cancer screening tests and cancer precursors. J Low Genit Tract Dis 2013; 17(5 Suppl 1):S1-27.
20. Han SN, Lotgerink A, Gziri MM, Van Calsteren K, Hanssens M, Amant F. Physiologic variations of serum tumor markers in gynecological malignancies during pregnancy: a systematic review. BMC Med 2012; 10:86.

21. Salani R, Billingsley CC, Crafton SM. Cancer and pregnancy: an overview for obstetricians and gynecologists. Am J Obstet Gynecol 2014; 211:7-14

22. Cappell MS. Colon cancer during pregnancy. The gastroenterologist's perspective. Gastroenterol Clin North Am 1998; 27:225-56.

23. Vieira R, Gomes R, Trajano A. Câncer de mama e gravidez subsequente: um olhar sociocultural. Rev Bras Cancerol 2005; 51:101-10.

24. Viellas EF, Domingues RMSM, Dias MAB, Gama SGN, Theme Filha MM, Costa JV, et al. Atenção pré-natal no Brasil. Cad Saúde Pública 2014; 30 Suppl:S85-100.

25. Torres JA, Domingues RMSM, Sandall J, Hartz Z, Gama SGN, Theme Filha MM, et al. Cesariana e resultados neonatais em hospitais privados no Brasil: estudo comparativo de dois diferentes modelos de atenção perinatal. Cad Saúde Pública 2014; 30 Suppl:S220-31.

26. Gama SGN, Viellas EF, Schilithz AOC, Theme Filha MM, Carvalho ML, Gomes KRO, et al. Fatores associados à cesariana entre primíparas adolescentes no Brasil, 2011-2012. Cad Saúde Pública 2014; 30 Suppl:S117-27.

27. Szwarcwald CL, Escalante JJC, Rabello Neto DL, Souza Junior PRB, Victora CG. Estimação da razão de mortalidade materna no Brasil, 2008-2011. Cad Saúde Pública 2014; 30 Suppl:S71-83.

28. Vega C, Castro Filho J, Pazero L, Caron O. Metodologia de coleta de casos de mortalidade materna no Município de São Paulo - 2001 a 2005. Saúde Soc 2008; 18 Suppl 1:79-80.

29. Soares VMN, Azevedo EMM, Watanabe TL. Subnotificação da mortalidade materna no Estado do Paraná, Brasil: 1991-2005. Cad Saúde Pública 2008; 24:2418-26.

30. World Health Organization. International statistical classification of diseases and related health problems, 10th revision. http://apps. who.int/classifications/icd10/browse/2015/en (acessado em 13/Mar/2016).

31. World Health Organization. The global strategy for women's, children's and adolescent's health (2016-2030): survive, thrive, transform. http:// www.who.int/life-course/partners/globalstrategy/en/ (acessado em 13/Mar/2016). 


\section{Abstract}

This population-based study included all the late maternal deaths from the case series of the Maternal Mortality Committees in the city of São Paulo and the state of Paraná, Brazil. The study compared 134 deaths identified by the São Paulo committee and 124 from the Parana committee in 2004-2013, aimed at demonstrating the magnitude and causes of late maternal mortality. Late maternal deaths accounted for $13.4 \%$ of all maternal deaths in the case series of the São Paulo committee and $12.1 \%$ in the cases series of the Parana committee. Direct obstetric causes accounted for 32.1\% of the late maternal deaths in São Paulo and $42.1 \%$ in Paraná, with postpartum cardiomyopathy as the principal cause in both case series. Death occurred between 43 and 69 days postpartum in 44\% of the cases in São Paulo and 39.5\% in Paraná. The correction factor for late maternal death was 3.3 in São Paulo and 4.3 in Paraná. Late maternal death had a relevant impact on overall maternal mortality, and postpartum cardiomyopathy was the principal cause of late direct obstetric death.

Maternal Mortality; Maternal Death; Cause of Death

\section{Resumen}

Estudio de base poblacional que incluyó todos los óbitos maternos tardíos de la casuística de los comités de mortalidad materna del municipio de São Paulo y del estado de Paraná, Brasil. Fueron comparados 134 óbitos del comité de São Paulo con los 124 de Paraná, durante el período 2004-2013, teniendo por objetivo demostrar la magnitud y las causas de mortalidad materna tardia. Los óbitos maternos tardios representaron un 13,4\% del total de los óbitos maternos en la casuística del comité de São Paulo y un 12,1\% en la de Paraná. Las causas obstétricas directas representaron un 32,1\% de los óbitos maternos tardios, según la casuística del municipio de São Paulo y un 42,1\% en Paraná, siendo la principal causa la cardiomiopatía pos-parto en las dos casuísticas. El óbito se produjo entre 43 y 69 dias tras el parto en un 44\% de los casos en São Paulo, y 39,5\% en Paraná. El factor de corrección para la muerte materna tardía fue 3,3 en la casuística de São Paulo y 4,3 en la de Paraná. La muerte materna tardía tuvo un relevante impacto sobre la mortalidad materna general y la cardiomiopatía pos-parto fue la principal causa de óbito obstétrico directo tardio.

Mortalidad Materna; Muerte Materna;

Causas de Muerte
Recebido em 02/Dez/2015

Versão final reapresentada em 29/Abr/2016

Aprovado em 30/Mai/2016 